\title{
IGNITION FEATURES OF PLASMA-BEAM DISCHARGE IN GAS-DISCHARGE ELECTRON GUN OPERATION
}

\author{
Valery A. Tutyk ${ }^{a, *}$, Anatoliy N. Ovcharuk ${ }^{b}$, Michail I. GasiK $^{c}$, \\ DMitriy V. MASLENIKOV ${ }^{d}$ \\ ${ }^{a}$ Department of physics, National Metallurgical Academy of Ukraine, Dnepropetrovsk, Ukraine \\ ${ }^{b}$ Department of electrometallurgy, National Metallurgical Academy of Ukraine, Dnepropetrovsk, Ukraine \\ ${ }^{c}$ Department of electrometallurgy, National Metallurgical Academy of Ukraine, Dnepropetrovsk, Ukraine \\ $d$ Department of physics, National Metallurgical Academy of Ukraine, Dnepropetrovsk, Ukraine \\ * corresponding author: tutykva@ua.fm
}

\begin{abstract}
The current paper presents the results of experimental researches to determine the mode features of plasma-beam discharge (PBD) generation by an electron beam injected by a low-vacuum gasdischarge electron gun (LGEG) with the cold cathode and hollow anode on the basis of the high-voltage glow discharge and in the range of helium pressure of $P \approx 10 \div 130 \mathrm{~Pa}$. The PBD boundaries and their dependences on parameters of an electron beam are found. The influence of PBD on parameters of low-vacuum gas-discharge electron gun is revealed. It causes an avalanche increase of electron beam current and burning of plasma-beam discharge in the whole space of the vacuum chamber volume and generation of electromagnetic radiation is revealed. Achieved results will be used for implementation of various vacuum technologies in the medium of reaction gas and generated electromagnetic radiation.
\end{abstract}

KEywords: plasma-beam discharge, vacuum, electron beam, electron gun, electromagnetic radiation.

\section{INTRODUCTION}

Perspectives of application of plasma-beam discharge (PBD) for creation of new electron beam and plasmachemical technologies is determined by high efficiency of beam electrons kinetic energy transformation into the HF (high-frequency) fields and plasma particles energy [12, 5, 1]. The phenomenon of beam instability is in the effective initiation of fluctuations and waves in plasma by means of electron beam, which came into being after publication of the fundamental works by Akhiyezer and Faynberg, Boma and Gross. This is used for the scientific issues and applicative purposes of the researches to investigate the operated thermonuclear synthesis, to find new methods of acceleration plasma, to work with plasma electronics, to carry out the experiments in the space etc. [2, 9, 4,

The use of PBD in plasma-chemical reactors with the purpose to receive chemically pure substances and implement the CVD and PCVD technologies on their basis for coatings and crystal growth in the reaction gas medium is of great practical interest [2]. This implementation is rational in the media of intermediate and low vacuum. PBD ignition at the pressure of neutral gas reaching the values of approximately $6650 \mathrm{~Pa}$ is theoretically proved [2]. Having been investigated out of the magnetic field and within the neutral gas pressure range of $\sim 0.05 \div 10 \mathrm{~Pa}$ by well known experimental works [7, 3, 6], PBD attracts practical, scientific and technical interest in the field of low pressure. For work in this range, low-vacuum gas-discharge electron guns (LGEG) with the hollow anode on the basis of the high-voltage glow discharge
(HVGD) applied to implement various electron beam technologies [10, 8, 11] is created. The present work studies the features of PBD formed by the electron beam which is injected with the low-vacuum gas discharge electron gun in the medium of low pressure neutral gas $(P \approx 10 \div 133 \mathrm{~Pa})$.

\section{Aims}

The aim of the work is experimental researches of features of plasma-beam discharge (PBD) generation mode in the gas discharge electron gun operation with cold cathode and the hollow anode on the basis of the high-voltage glow discharge in a range of helium pressure $P \approx 10 \div 130 \mathrm{~Pa}$.

\section{Materials And Methods}

Experimental studies of PBD created by LGEG, were carried out on the plant, which scheme is shown in Fig. 1 .

LGEG (1) is fed from the high-voltage power supply (2) of direct current of $5 \mathrm{~kW}$. The parameters are the following: with accelerating voltage of $0 \div 20 \mathrm{kV}$ and the high-voltage pulse generator forming tension impulses with amplitude of $1 \div 40 \mathrm{kV}$, time of $\sim 10 \mu$ s and repetition frequency of $50 \div 100 \mathrm{~Hz}$ were used. High voltage of negative polarity is introduced to LGEG cold aluminum cathode. The anode made from stainless steel is earthed on the case of the vacuum chamber (6).

Measurement of anode current $I_{\mathrm{A}}$ is carried out by means of the $R_{3}$ resistor. The current of an elec- 

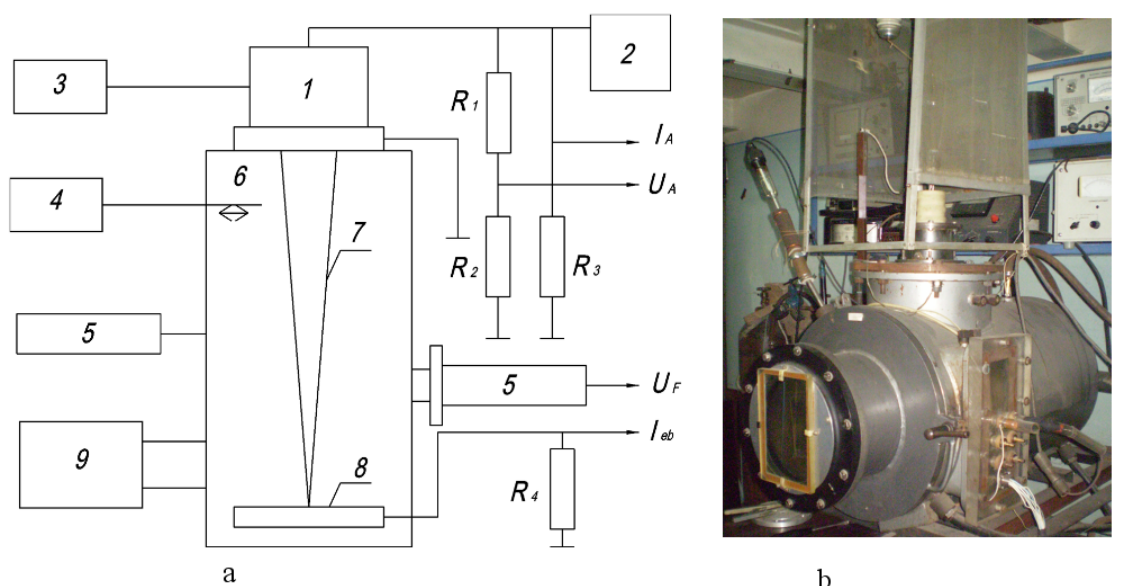

$\mathrm{b}$

Figure 1. Schematic diagram (a) and general view (b) of the experimental plant; 1 - low-vacuum gas discharge electron gun, 2 - power supply, 3 - system of blowing, 4 - double probe, 5 - photo-electronic multiplier of the FEU-19 type, 6 - case of the vacuum chamber, 8 - collector, 9 - vacuum pump.

tron beam $I_{\mathrm{E}}$ coming to the collector (8) is measured from $R_{4}$ resistor. High voltage on the cathode determined by the voltage divider $R_{1}, R_{2}$. LGEG is located on a dielectric flange. Inside of the vacuum chamber (6) the dynamic vacuum is supported. Evacuation is formed by the vacuum pump (9), while the working gas of helium is continuously blown from the blowing system (3) into the area of HVGD gun. Studies are made when helium pressure is in a range of $10 \div 133 \mathrm{~Pa}$. Measurement of EB current distribution on the cross section is performed with the use of "the hole chamber" (Faradey's screened cylinder). Parameters of plasma are measured by a double electric probe, which could move transversely of the vacuum chamber. During the operation in the pulse mode, the following EB parameters are measured by oscillographic method: current, accelerating voltage, pulse type, etc. The radiation of light from the area where EB interacted with neutral gas and plasma is recorded by means of the photo-electronic multiplier (5) of the FEU-19 type.

In the experimental research, low-voltage gas-discharge guns with hollow anode and cold cathode of EGP-9 type (Fig. 2), [11] are used, including the main components shown below.

LGEG operation can be described by the following. After giving of high accelerating voltage $U_{\mathrm{A}}$ to the electrodes of LGEG (Fig. 3) HVGD is ignited along the axial line of the anode opening. The formed ions $\mathrm{i}^{+}$ start to bombard the cold cathode and as a result of $\gamma$-processes beam fast electrons $\mathrm{e}_{\mathrm{b}}^{-}$appear. The latter during their movement create the new ions $\mathrm{i}^{+}$ and slow electrons $\mathrm{e}_{\mathrm{m}}^{-}$due to the collisions with neutral particles $\mathrm{A}=\mathrm{i}^{+}+\mathrm{e}_{\mathrm{m}}^{-}$.

The new-formed ions are partly grasped by electric field and move to the cathode. On their way some of them undergo recharging to cause fast neutral particles (A) formation, which continue their movement to the cathode. Due to the cathode bombing

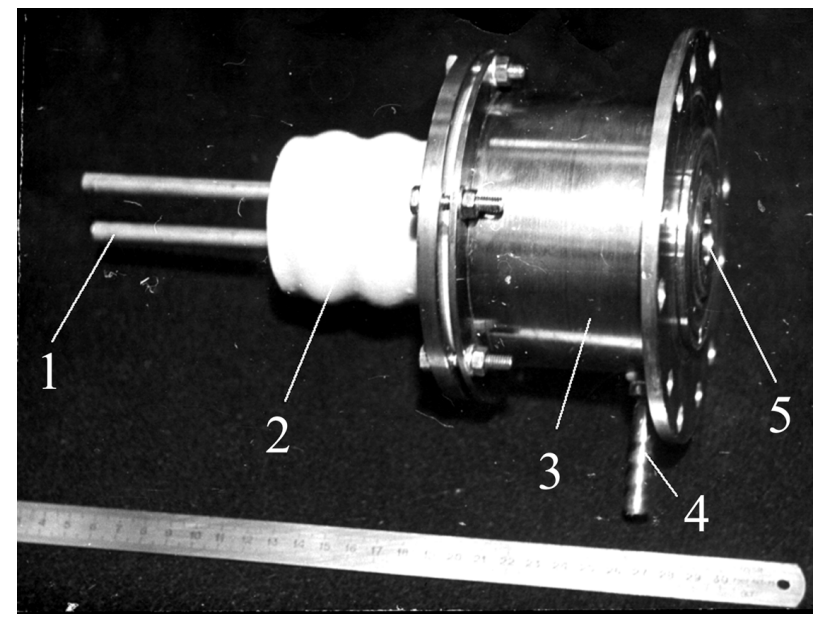

FIgURE 2. General view of LGEG of EGP-9 type; 1 - cathode water-cooled joint, 2 - high-voltage ceramic insulator, 3 - hollow anode block, 4 - elements of gas blowing system, 5 - replaceable anode.

by ions $\mathrm{i}^{+}$and fast particles, new fast electrons $\mathrm{e}_{\mathrm{b}}^{-}$ come into being and the process repeats.

Leaving HVGD plasma, the electron beam (EB) creates. The other part of ions free from towards - cathode traction is attracted by EB to perform its ionic focusing. Slow electrons $\mathrm{e}_{\mathrm{m}}^{-}$are drawn to the anode and compensate the charge of the fast electrons $\mathrm{e}_{\mathrm{b}}^{-}$from the beam, which have left LGEG, allowing the gun to work without the electron collector.

\section{Results AND Discussion}

Experimental researches have shown that LGEG performance is characterized by the two modes of work:

(1.) ion focusing mode (Fig. 4 a 4 c); and

(2.) plasma-beam discharge mode (Fig. 4 $\mathrm{b}-4 \mathrm{~d}$ ).

In small currents, the mode of ionic focusing (Fig. 4a) is observed and the presence of the ions 


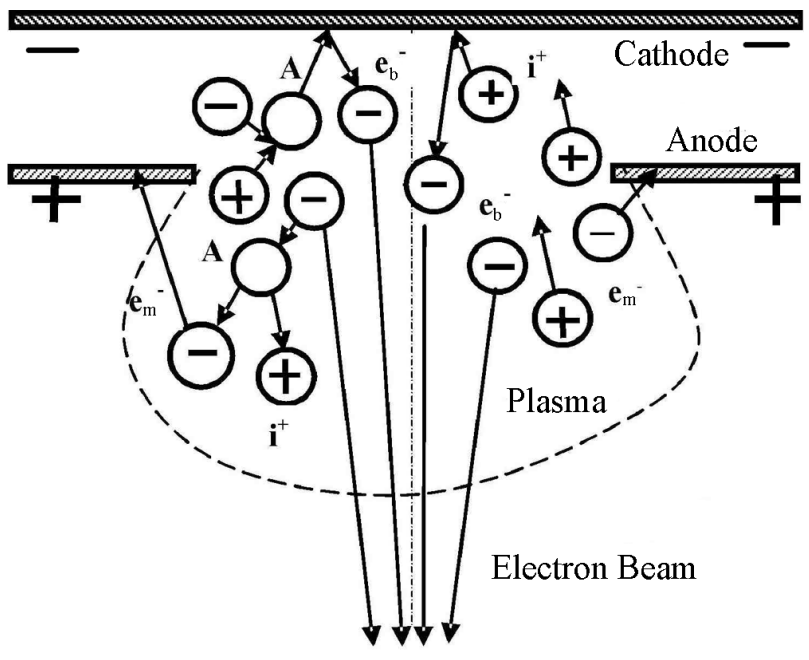

FIGURE 3. Schematic of LGEG perfomance.
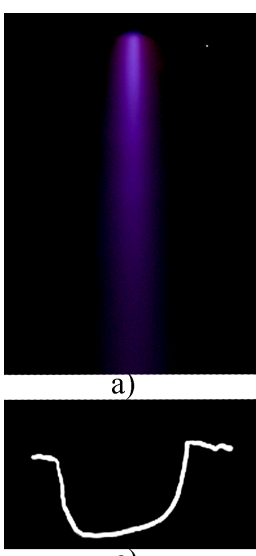

c)
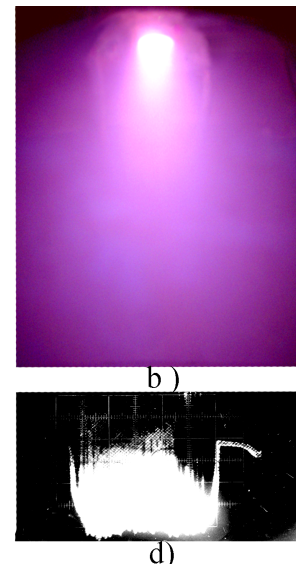

Figure 4. LGEG modes.

focused by EB ions and created by impact ionization, is its main feature. The luminescence of the longitudinal crossover of EB in helium is blue, that can be related to high energy of electron beam $\left(U_{\mathrm{A}} \sim 1 \div 10 \mathrm{kV}\right)$, which makes possible actuation the highest levels of helium atoms and ions. The form of current pulse (Fig. 4.) indicates the absence of fluctuations in system. The increase in EB current higher than the limited one (at $P=$ const) causes ignition of $\mathrm{PBD}$ (Fig. 4b). Thus plasma with a pink luminescence fills all the internal space of the vacuum chamber. Change in luminescence color towards the longer waves means considerable decrease in energy of electrons participating in actuation and ionization of neutral gas atoms. Ignition of PBD in the pulse mode leads (Fig. 4d) to formation of current pulse of HF fluctuation at the top.

As researches showed, the PBD peculiarity is that the discharge ignition takes place in the whole internal space of vacuum chamber when LGEG work while ignition under the same conditions but without the magnetic field [7, 6] is characterized by plasma formation around the electron beam. This essential difference can be explained by as bel-

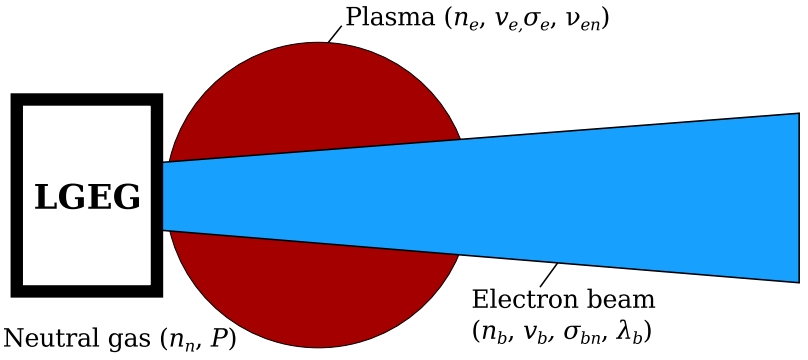

Figure 5. Condition of PBD ignition.

low. The electron beam, injected by LGEG interacts both with own plasma, created by itself with impact ionization, and with HVGD plasma, which develops this electron beam (Fig. 5). Analyzing the results of PBD formation mechanism in LGEG operation, it is possible to report on the following. Having certain parameters of EB and neutral gas pressure, the HVGD plasma concentration $n_{\mathrm{e}}$ and concentration of electrons in beam $n_{\mathrm{b}}$ reach the required value to develop plasma beam instability and PBD ignition.

However, PBD ignition provokes increase in plasma density in HVGD because of the neutral gas atoms additional ionization by electric fields of flame fluctuation. This is accompanied with the increase of ions which are bombarding the cathode. The avalanche increase of EB current occurs. Then the processes are being intensified and PBD fills the whole internal space of vacuum chamber where $\mathrm{EB}$ is injected. The most possible explanation is by the relation to the positive feedback which appears between EB and PBD. It intensifies the processes of EB energy transformation into the energy of PBD. Thus, due to this feedback, in PBD mode, LGEG creates the plasma formation which is considerably bigger in size. The similar formation is created by thermionic guns as it fills the working volume of the vacuum chamber.

The experimental researches determine the boundaries of PBD ignition at various pressure $P$ depending on current $I=f(P)$ (Fig. 6) and EB capacity $N=\Theta(P)$ (Fig. 7) with the varying diameters of anode hole $d_{\mathrm{A}}$. The graphs curves 1 are measured at $d_{\mathrm{A} 1}=10 \mathrm{~mm}$, and for curves 2 at $d_{\mathrm{A} 2}=8 \mathrm{~mm}$. Increase of pressure cause increase of the current and EB capacity, these conditions are required for PBD ignition. Its regular character can be explained in such a way. It is well known [2], that the necessary conditions for beam instability development are the following:

(1.) size of plasma $d$ is not to exceed the length of free run of beam electrons $\lambda_{\mathrm{b}}$ in the gas,

$$
d \leq \lambda_{\mathrm{b}}
$$

(2.) the energy of electron beam is to be efficiently delivered to the plasma 2 when increment of beam instability,

$$
\delta=\omega_{\mathrm{pe}}\left(\frac{n_{\mathrm{b}}}{n_{\mathrm{e}}}\right)^{1 / 3}>5 \cdot \nu_{\mathrm{en}},
$$




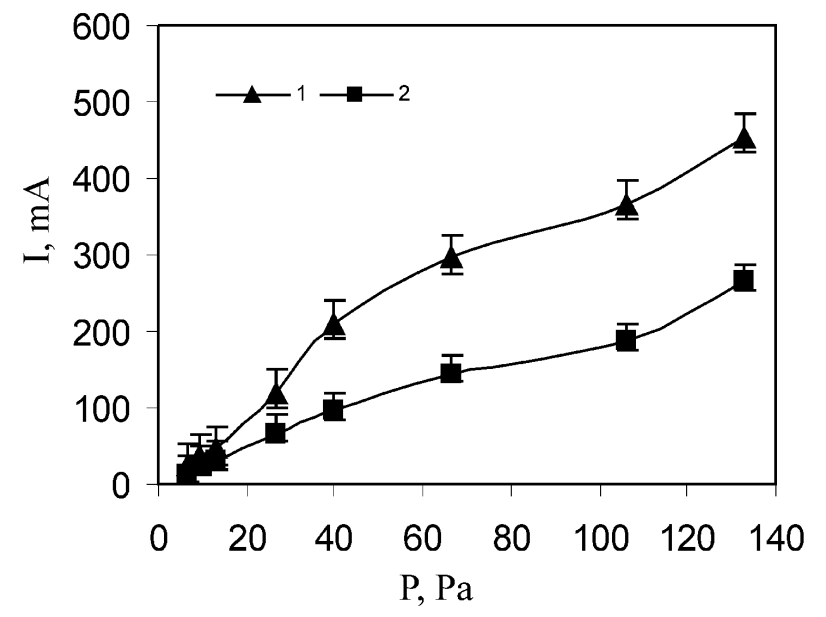

Figure 6. Dependence of EB limit current for PBD ignition on helium pressure.

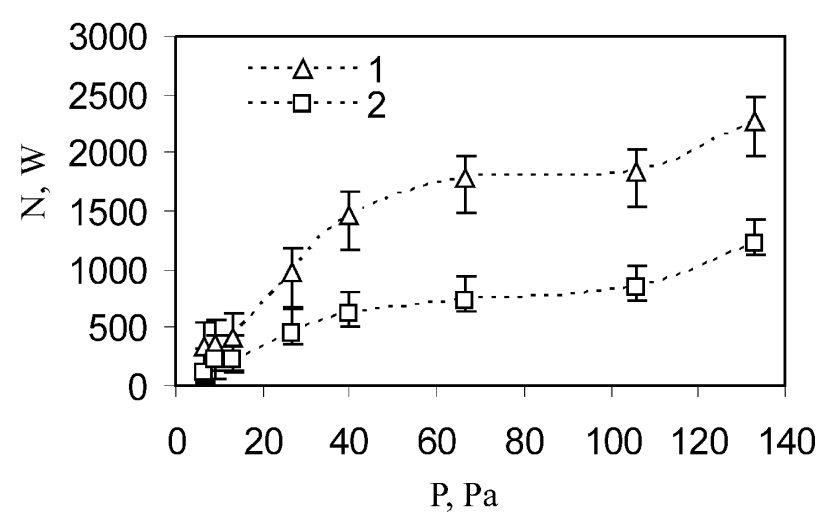

Figure 7. Dependence of EB limit power required for PBD ignition on helium pressure.

where $\nu_{\mathrm{en}}$ is collision frequency of plasma electrons with neutrals, $\omega_{\text {pe }}=5.64 \times 10^{4} n_{\mathrm{e}}^{1 / 2}$ is Langmuire electron frequency of plasma. This phenomenon is related to the fact that PBD in a certain sense is analog of a classic HF discharge, where HF waves $\left(\omega \gg \nu_{\mathrm{en}}\right)$ are actuated by electron beam [4].

The increase in pressure leads to increase in concentration of neutral particles $n_{\mathrm{n}}=P / k T$, (where $k$ is Boltzmann constant and $T$ is gas temperature). This increases in collision frequency of plasma electrons with neutrals $\nu_{\mathrm{en}}=3.4 \cdot 10^{7} \cdot a^{2} \sqrt{T} \cdot n_{\mathrm{n}} \mathrm{s}^{-1}[3$, where $a=0.95 \times 10^{-8} \mathrm{~cm}$ is an efficient radius of helium atom, $T$ is temperature of plasma electrons or energy of electrons $(\mathrm{eV})$ and condition Eq. 2 of PBD ignition fails because $\nu_{\mathrm{en}}$ increased. It is necessary to increase current and EB power in order to restore it.

Main influence on PBD ignition renders neither to the current value nor to EB capacity, but to electron concentration $n_{\mathrm{b}}$. In the Fig. 8 the dependences of critical EB concentration in PBD ignition from helium pressure for various diameters $d_{\mathrm{A} 1}=10 \mathrm{~mm}$ and $d_{\mathrm{A} 2}=8 \mathrm{~mm}$ is shown. Decrease of the beam diameter in constant flow to the decrease of anode hole diameter results in increasing in electron concen-

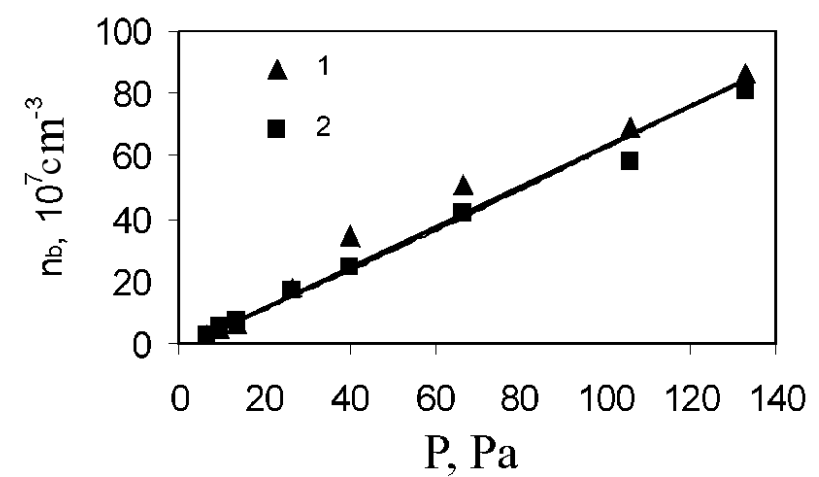

FiguRE 8. Dependence of EB critical concentration in PBD ignition on helium pressure.

tration in the beam and, as Figs. 6, 7 and 8 illustrate, decrease in both EB flow and power, is the necessary condition for PBD ignition.

Let's consider the correspondence of the experimentally obtained conditions of PBD ignition to the theoretically developed ones. Optical measurements, done by photographing the area of PBD burning near the anode hole, evidence the fulfillment of the condition Eq. 1 in the whole range of LGEG operating pressure. So, for example, when EB with $U_{\mathrm{A}}=5000 \mathrm{~V}$ at $P_{1}=13 \mathrm{~Pa}$, the measured values $d_{1} \sim 8 \mathrm{~cm}$, $\lambda_{\mathrm{b} 1} \geq 36 \mathrm{~cm}$; when $P_{2}=100 \mathrm{~Pa}-d_{2} \sim 3 \mathrm{~cm}$, $\lambda_{\mathrm{b} 2}=13 \mathrm{~cm}$ and correspondingly in both cases $d<\lambda_{\mathrm{b}}$. Then we estimate condition of PBD ignition described by Eq. (2). The data provided by double probe measurement are as follows: for $P_{1}=13 \mathrm{~Pa}, I_{\mathrm{b} 1}=0.1 \mathrm{~A}$, $U_{\mathrm{A} 1}=5000 \mathrm{~V}$ value of $\nu_{\mathrm{en} 1} \sim 10^{7} \mathrm{~s}^{-1}$. Concentration of plasma electrons $n_{\mathrm{e} 1} \sim 10^{10} \mathrm{~cm}^{-3}$, when electron Langmuire frequency of plasma is

$$
\omega_{\mathrm{e} 1}=5.64 \times 10^{4} \sqrt{n_{\mathrm{e} 1}} \approx 6 \times 10^{9} \mathrm{~s}^{-1} .
$$

Value of $n_{\mathrm{b} 1} \sim 2 \times 10^{8} \mathrm{~cm}^{-3}$ (for anode hole $d_{\mathrm{A}}=10 \mathrm{~mm}$ ). Value of increment of beam instability is expressed by

$$
\delta_{1}=\omega_{\mathrm{e} 1}\left(n_{\mathrm{b} 1} / n_{\mathrm{e} 1}\right)^{1 / 3} \approx 2 \times 10^{9} \mathrm{~s}^{-1} .
$$

It become obvious, that condition Eq. 2 is true as indicates the possibility of PBD ignition with these parameters. Let's consider a case with higher pressures, when $P_{2}=133 \mathrm{~Pa}, I_{\mathrm{b} 2}=0.5 \mathrm{~A}, U_{\mathrm{A} 2}=5000 \mathrm{~V}$, when plasma parameters are as follows: value of $\nu_{\mathrm{en} 2} \sim 10^{8} \mathrm{~s}^{-1}$; concentration of electron of a beam is $n_{\mathrm{b} 2} \sim 7 \times 10^{8} \mathrm{~cm}^{-3}$; concentration of electrons of plasma made $n_{\mathrm{e} 2} \sim 3 \times 10^{11} \mathrm{~cm}^{-3}$; electron Langmuire frequency of plasma $\omega_{\mathrm{e} 2} \approx 3 \times 10^{10} \mathrm{~s}^{-1}$. Taking into account the presented parameters, the value can be expressed as here:

$$
\delta_{2}=\omega_{\mathrm{e} 2}\left(n_{\mathrm{b} 2} / n_{\mathrm{e} 2}\right)^{1 / 3} \approx 10^{11} \mathrm{~s}^{-1} .
$$

Condition Eq. 2 for this case is also true as well and PBD is ignited, that corresponds to experimental measurements. 
Results achieved by the present work prove that it is possible to have PBD with the use of LGEG under the pressure of $P \approx 10 \div 133 \mathrm{~Pa}$. The phenomenon is accompanied by the plasma formation where electron concentration is $n_{\mathrm{e}} \approx 10^{10} \div 10^{11} \mathrm{~cm}^{-3}$, and occupies the whole working volume of the vacuum chamber. The existence of EB, HF-fields, plasma of large volume and low vacuum make it possible to implement new CVD and PCVD technologies under such conditions in the area of reactionary gas to receive the coatings, grow crystals and for the needs of various technologies of plasma chemistry.

\section{Conclusions}

The present work proves experimentally that the two working modes of LGEG operation with pressures of $P \approx 10 \div 133 \mathrm{~Pa}$ are possible. They are the mode of ion focusing and the mode of plasma-beam discharge. Transition from one mode to another is determined by the parameters of an electron beam and gas pressure.

Areas of PBD ignition with elevated helium pressure $(P \approx 10 \div 133 \mathrm{~Pa})$ are identified within dependence on current, capacity and concentration of EB electrons. It is shown that the increase in pressure leads to the necessity to increase both current and EB capacity in order to ignite PBD. However, the determining factor is the increase of electron concentration in $\mathrm{EB}$ which change in the range of $n_{\mathrm{b}} \approx 10^{7} \div 10^{8} \mathrm{~cm}^{-3}$.

The current researches of the presented work find out the influence of PBD on LGEG operation. This results in avalanche increase of EB current and PBD ignition which take the space of the whole working volume of the vacuum chamber where EB is injected. The measurements of concentration of PBD plasma electrons were carried out under conditions of increasing pressure and they showed that electrons concentration was within the range of $n_{\mathrm{e}} \approx 10^{10} \div 10^{11} \mathrm{~cm}^{-3}$.

Generation of large volume of plasma in LGEG operation when it works in a PBD mode is a perspective direction for implementation of various CVD and PCVD technologies in the medium of reaction gas. Transition from one mode to another is defined by parameters of electron beam and gas pressure.

\section{REFERENCES}

[1] A. I. Akhyiezer, L. A. Akhyiezer, R. V. Polovin, et al. Electrodynamics of plasma. Nauka, Moscow, 1974. (in Russian).

[2] A. A. Ivanov, V. G. Leiman. O zagiganii plazmenno-puchkovogo razriada moschnym electronnym puchkom v gase bolshoj plotnosti. Physics of plasma 3(4):780-785, 1977. (in Russian).

[3] E. A. Kornilov, et al. Losses of energy of beam in vapor-gas channel. Physics and chemistry of treatment of materials 2:20-23, 1975. (in Russian).

[4] V. E. Mishin, Yu. A. Ruzhin, V. A Telegin. Interaction of electron beams with ion-sphere plasma. Hydrometeoizdat, Leningrad, 1989. (in Russian).

[5] V. I. Perevodchikov. Beam-plasma processes in electron-beam apparatus of industrial application. In $S b$. nauch. tr. VEI. VEI, Moscow, 1994. (in Russian).

[6] V. P. Popovych, et al. Investigation of condition of formation of plasma-beam discharge without magnetic field. Radiophysics 16(6):1109-1117, 1973. (in Russian).

[7] V. P. Popovych, I. F. Kharchenko, E. G. Shustin. Beam-plasma discharge without magnetic field. Radiotechnika i electronika 18(3):649-651, 1973. (in Russian).

[8] Yu. Ya. Ruzhin, V. N. Oraevsky, V. A. Tutyk. The active experiments in the stratosphere with the electron beams injection. Adv Space Res 13(10):(10)117-(10)122, 1993.

[9] V. T. Tolok. Physics of plasma and problems of controlled thermonuclear synthesis. Respubl. mezvedomstv. sb. Naukova dumka, Kyiv, 1971. (in Russian).

[10] V. A. Tutyk. Effect of electron run away in electron gas-discharge guns with hole anode. In "Vacuum science and engineering" Proceedings of X. scientific-engineering conference in 2 volumes, pp. 458-463. MIEM, Moscow, 2003. (in Russian).

[11] V. A. Tutyk. Gas-discharge electron guns for aerodynamics investigations. In Collection of reports of 7-th International conference "Vacuum nanotechnologies and equipment", pp. 50-54. NNC KPTI, "Constanta", Kharkiv, 2006. (in Russian).

[12] M. A. Zavialov, Yu. E. Kreindel, A. A. Novikov, L. P. Shanturin. Plasma processes in technological electron guns. Energoatomizdat, Moscow, 1989. (in Russian). 